\title{
Changes in the Olfactory Response to Amino Acids in Japanese Newts After Transfer from an Aquatic to a Terrestrial Habitat
}

\author{
Ritsuko Inoue and Kei Nakatani ${ }^{*}$ \\ Graduate School of Life and Environmental Sciences, University of Tsukuba, \\ 1-1-1 Tennoudai, Tsukuba, Ibaraki 305-8572, Japan
}

\begin{abstract}
Amphibians are capable of smelling both volatile and water-soluble (e.g., amino acids) odorants. Adult Japanese newts, Cynops pyrrhogaster, live mostly in water, except during hibernation, but sometimes on land. To examine olfactory responses of the newts to adaptation to a short-term stay on land (land adaptation), we measured the magnitude of the olfactory response at five different time points (land adaptation time: $0,30,54,90$, and $114 \mathrm{~h}$ after transfer from an aquatic to a terrestrial habitat by using electro-olfactogram (EOG) recordings. Statistical analysis by the weighted linear model $(P<0.05)$ indicated that the time to land adaptation had a significant effect on the magnitude of the EOG induced by $1 \mu \mathrm{M}$ and $10 \mu \mathrm{M}$ amino acid mixtures. Further, the slope estimates of the weighted linear model were significantly positive $(P<0.05)$. These results indicate that the magnitude of the EOG response to amino acid mixtures (arginine, alanine, proline, and glutamic acid) significantly increases with land adaptation time. On the other hand, we observed no significant relationship between the magnitude of the EOG response induced by an $0.05 \%$ volatile odorant mixture (isoamyl acetate, $\mathrm{n}$-amyl acetate, cineole, and limonene) and land adaptation time. Our results indicate that olfactory sensitivity to amino acids significantly increases with land adaptation time in adult Japanese newts.
\end{abstract}

Key words: olfaction, olfactory response, newt, electro-olfactogram, amino acid odorant

\section{INTRODUCTION}

Odorants include volatile and water-soluble odorous substances. Volatile substances are well-known odorants, and their detection by animals is dependent on the habitat of the animals. Terrestrial vertebrates, including humans, use their olfactory system to detect only volatile odorants, while aquatic vertebrates such as fish use their olfactory system to detect only water-soluble odorants (Hara, 1994). Amino acids are water-soluble odorants. Amino acids are used as feeding stimuli by several species of aquatic predators, such as giant tiger prawn (Coman et al., 1996) and some teleosts (Hara, 2006).

Amphibians have been used as model animals in studies of vertebrate olfactory sense (e.g., Nakamura and Gold, 1987; Kurahashi, 1990). Olfactory epithelia in vertebrates contain olfactory receptor neurons (ORNs), supporting cells, and basal cells. The process of olfaction begins with the activation by odorants of olfactory receptors (ORs) located in the olfactory cilia (Buck and Axel, 1991). The unique olfactory response of amphibians has been studied extensively. Amphibians have adapted to both terrestrial and aquatic life. Early behavioral studies suggest that amphibians are capable of olfaction of both volatile and water-

\footnotetext{
* Corresponding author. Phone: +81-298-53-6672; Fax : +81-298-53-6614; E-mail: nakatani@biol.tsukuba.ac.jp
}

soluble odorants (Reese, 1912; Copeland, 1913). Recent molecular studies on ORs have shown amphibians to have two classes of ORs, class-I receptors for water-soluble odorants and class-II receptors for volatile odorants (Freitag et al., 1998; Mezler et al., 2001). Yamada and Nakatani (2001) used patch-clamp recordings to demonstrate that ORNs in the Japanese newt, Cynops pyrrhogaster, respond to both volatile odorants and amino acids.

The unique biological characteristics of amphibians correlate to changes in their olfactory responses. Olfactory response characteristics change during metamorphosis in the tiger salamander (Arzt et al., 1986). Nakazawa et al. (2000) reported oscillatory potential changes in the olfactory epithelium of the Japanese toad during the breeding period. There are species-specific differences in the lifestyle of adult amphibians. Adult Japanese newts live mostly in water, except during hibernation, but sometimes on land. When newts are transferred from water to land, they begin to grow olfactory cilia (Shibuya and Takagi, 1963). After a short-term stay on land, the olfactory system may adapt to the terrestrial lifestyle. It is not completely understood, however, how adaptation to a short-term stay on land (land adaptation) influences the olfactory response of the newts.

We employed electro-olfactogram (EOG) recordings to measure the magnitude of the olfactory response, and examined the effects of land adaptation on the olfactory response by fitting the data to a weighted linear model. Our results indicate that olfactory sensitivity to amino acids significantly increases with land adaptation time. 


\section{MATERIAL AND METHODS}

\section{Animals and preparation}

Adult male Japanese newts, Cynops pyrrhogaster, were purchased from a local animal supplier. The animals were kept in a tank filled about $10 \mathrm{~cm}$ deep with water at a temperature of about $20^{\circ} \mathrm{C}$. They were fed pellet food twice a week, except on the day before the experiments. In order for the animals to adapt to a terrestrial habitat, we transferred them from the water tank to a plastic container with no water (land adaptation). They were kept in the plastic container, in which the humidity was maintained to prevent them from drying, for 30-114 $\mathrm{h}$, depending on the experiment.

\section{EOG recording}

The newts were pithed and decapitated. The olfactory epithelium was exposed by cutting the roof of the nasal cavity. Procedure for recording EOG responses to test solutions was similar to that described in Shibuya and Takagi (1963). A glass pipette electrode with a tip diameter of $0.1 \mathrm{~mm}$ was used to record EOG. The pipette was filled with normal saline $(110 \mathrm{mM} \mathrm{NaCl}, 2.5 \mathrm{mM} \mathrm{KCl}, 1.0 \mathrm{mM}$ $\mathrm{CaCl}_{2}, 1.6 \mathrm{mM} \mathrm{MgCl} 2,5 \mathrm{mM}$ Na-HEPES; $\mathrm{pH}$ 7.6) and connected through an $\mathrm{Ag} / \mathrm{AgCl}$ wire to a conventional amplifier. The electrode was placed in the center of the olfactory epithelium. The head of the animal was mounted on a recording chamber, and solutions flowed continuously through an application tube into the chamber. Solutions were applied by gravity into a four-way valve that was operated pneumatically under remote control by computer. Normal saline was applied continuously during the experiments except when test solutions were applied. All recordings were performed at room temperature.

\section{Test solutions}

Two kinds of odorous fluids (water-soluble and volatile) were used as test solutions. One was an amino acid mixture containing four amino acids (arginine, alanine, proline, and glutamic acid) each at a concentration of $1 \mu \mathrm{M}$ or $10 \mu \mathrm{M}$. The other was a volatile odorant mixture containing four arbitrary volatile odorants (isoamyl acetate, $\mathrm{n}$-amyl acetate, cineole, and limonene). Each of the volatile odorants was dissolved in DMSO and used at a final concentration of $0.05 \%$. All test solutions were dissolved in saline. The $\mathrm{pH}$ of all solutions was adjusted to 7.6 .

\section{Statistical analysis}

To examine the effects of land adaptation, weighted linear modeling was performed using weighted least squares (Quinn and Keough, 2002). The variance of the magnitude of the EOG varied for different time points $(0,30,54,90$, and $114 \mathrm{~h})$. Therefore, we weighted each observation by the reciprocal of an estimate of its variance. We compared the two slope estimates (Table 1) by Student's $t$-test to determine differences between the magnitude of the EOG response to the amino acid mixture at concentrations of $1 \mu \mathrm{M}$ and $10 \mu \mathrm{M}$. We compared the intercept estimates to test solutions (Table 1) also by $t$-test, and determined whether the magnitude of the EOG response at $0 \mathrm{~h}$ was different for the odorants. Signifi-

Table 1. Estimated parameters for the weighted linear model. ${ }^{*}, P<$ 0.05 .

\begin{tabular}{cccccc}
\hline Test odorant & Estimate & SE & \multicolumn{1}{c}{$t$} & $\mathrm{P}$ \\
\hline $1 \mu \mathrm{M}$ amino acid mix & Intercept & 431.326 & 40.176 & 10.74 & $<0.001^{*}$ \\
& Slope & 2.703 & 1.06 & 2.55 & $0.0151^{*}$ \\
$10 \mu \mathrm{M}$ amino acid mix & Intercept & 429.294 & 88.99 & 4.824 & $<0.001^{*}$ \\
& Slope & 7.573 & 1.798 & 4.212 & $<0.001^{*}$ \\
Volatile odorant mix & Intercept & 590.56 & 80.89 & 7.3 & $<0.001^{*}$ \\
& Slope & 1.99 & 1.64 & 1.213 & 0.233 \\
\hline
\end{tabular}

cance was determined at $P<0.05$. All statistical analyses were conducted using the statistical package R (version 2.7.0, http://www.rproject.org/).

\section{RESULTS}

To examine the effects of terrestrial adaptation, we recorded the EOG response, an extracellular field potential where the amplitude reflects the summed response of individual ORNs (Ottoson, 1956). The maximal EOG response was referred to as the magnitude of EOG (indicated by arrows in Fig. 1). This method allowed us to simultaneously measure the activities of many cells in the olfactory epithelium.

In preliminary studies, we measured the amplitude of EOG responses to test solutions among different animals kept in the terrestrial habitat in increments of six hours from 6 to $126 \mathrm{~h}$, to obtain a rough time course of amplitude change (data not shown). On the basis of this result, we determined five different time points (land adaptation time: $0,30,54,90$, and $114 \mathrm{~h}$ after transfer from the aquatic to the terrestrial habitat) at which to record EOG responses.

Fig. 1 shows the EOG response to odorants by newts kept in the terrestrial habitat for $0,30,5490$, and $114 \mathrm{~h}$. For each recording, an electrode was placed on the olfactory epithelium on one side, and test solutions were applied to the same side. The application of test solutions to the olfactory epithelium evoked slow potentials (amino acid mixture, Fig. 1A; volatile odorant mixture, Fig. 1B). The magnitude of the EOG was greater at later time points.

To perform statistical analyses, we recorded the amplitude of the EOG response to odorants from at least four samples at each time point. Fig. 2 shows the mean magnitude of EOG to odorants plotted against land adaptation time. The magnitude of the EOG (mean \pm SE) induced by the $1 \mu \mathrm{M}$ amino acid mixture at each time point was 423.62 \pm 40.61, 952.61 \pm 300.06, 577.30 \pm 103.12, 800.13 \pm 156.97 , and $608.01 \pm 157.34 \mu \mathrm{V}$, respectively (Fig. $2 \mathrm{~A}$ ). Statistical analysis indicated that the time to land adaptation had a significant effect on the magnitude of the EOG (weighted linear model; $F_{1,37}=6.5002, P=0.01506$ ). Further, the slope estimate of the weighted linear model was significantly positive (slope estimate $=2.703, t=2.55, P=$ 0.0151 ; Table 1). These results indicate that the magnitude of the EOG response to the $1 \mu \mathrm{M}$ amino acid mixture significantly increased with land adaptation time. Similarly, the magnitude of the EOG induced by the $10 \mu \mathrm{M}$ amino acid mixture was significantly influenced by the time course of land adaptation (weighted linear model: $F_{1,37}=17.737, P<$ 0.001 ) and significantly increased with land adaptation time (slope estimate $=7.573, t=4.212, P<0.001$ ) (Table 1). The magnitude of the EOG (mean \pm SE) induced by the $10 \mu \mathrm{M}$ amino acid mixture at each time point was $397.91 \pm 94.54$, $899.30 \pm 242.71,897.56 \pm 147.09,1007.60 \pm 149.83$, and $1780.95 \pm 625.22 \mu \mathrm{V}$, respectively (Fig. $2 \mathrm{~B}$ ). Comparison of the two slope estimates from the weighted linear model showed a significant effect ( $t$-test; $P<0.05$ ), indicating that the increase in the magnitude of the EOG with land adaptation time was significantly greater for exposure to the 10 $\mu \mathrm{M}$ amino acid mixture than to the $1 \mu \mathrm{M}$ amino acid mixture.

Fig. 2C shows the mean magnitude of EOG induced by 
A

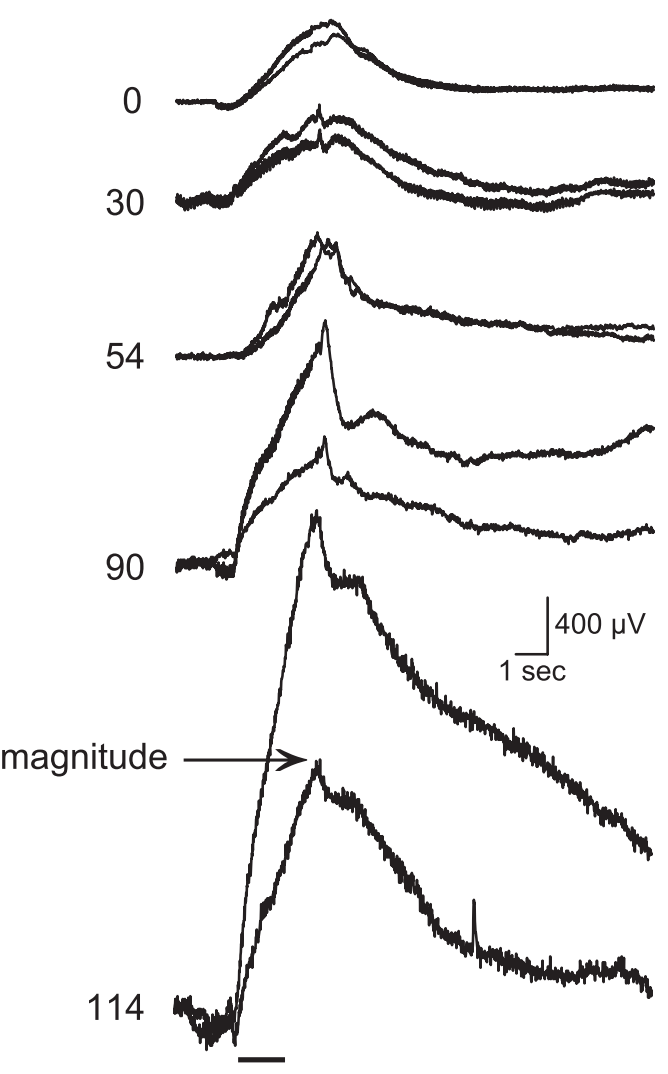

B

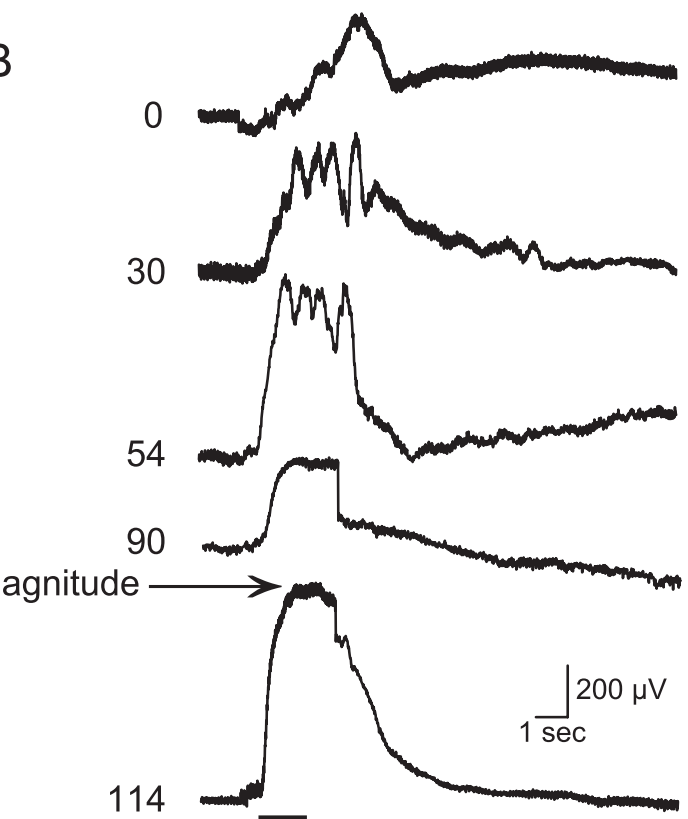

Fig. 1. Olfactory responses of Japanese newts transferred from an aquatic to a terrestrial habitat (land adaptation). (A) EOG response to an amino acid mixture containing four amino acids (arginine, alanine, proline, and glutamic acid). In these figures, the amino acid mixture $(1 \mu \mathrm{M}$ or $10 \mu \mathrm{M})$ was applied to the animal at each time point (land adaptation time: $0,30,54,90$, and $114 \mathrm{~h}$ ). The higher response in each recording is the EOG response to the $10 \mu \mathrm{M}$ amino acid mixture. (B) EOG responses to the volatile odorant mixture (isoamyl acetate, n-amyl acetate, cineole, and limonene). The numbers on the left indicate the land adaptation time. The bars indicate the time of application of the test solutions.

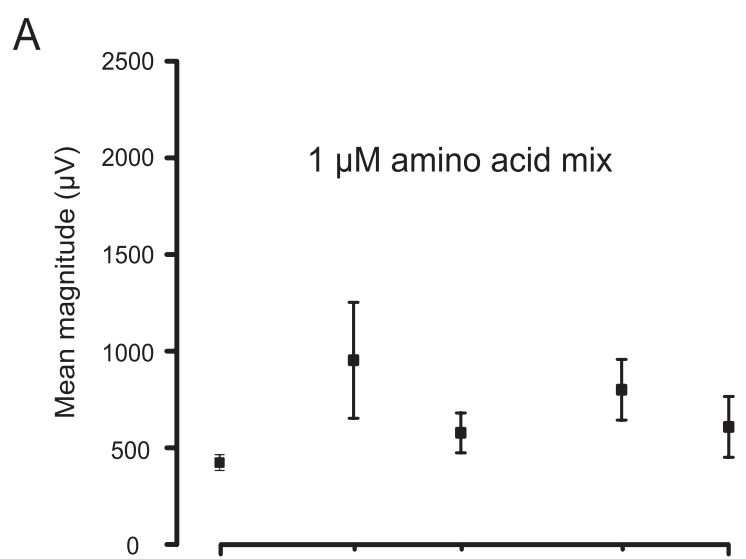

B

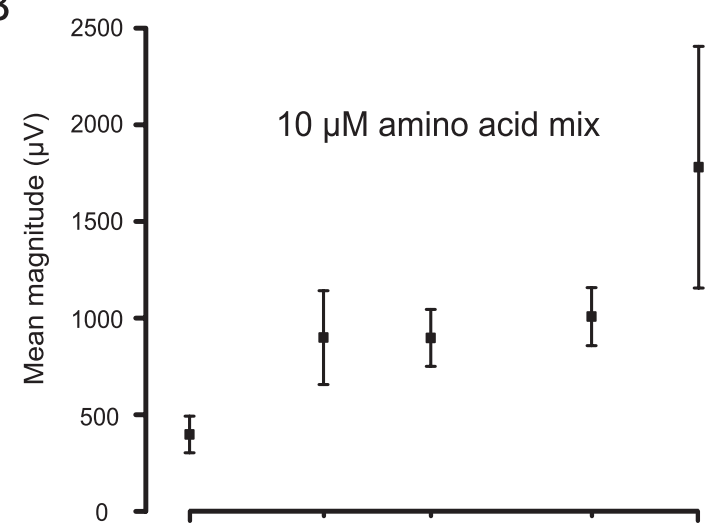

C

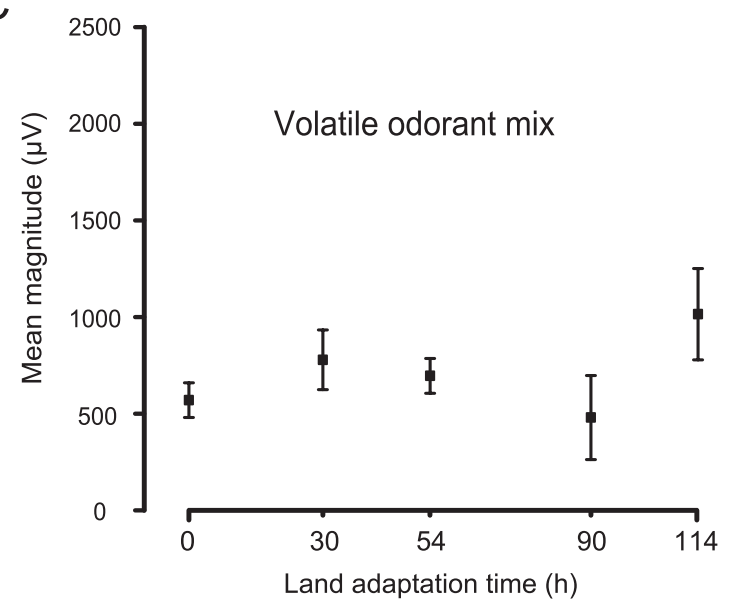

Fig. 2. Mean magnitude of the EOG to test odorants at land adaptation times of $0,30,54,90$, and $114 \mathrm{~h}$. The mean magnitude of the EOG to (A) a $1 \mu \mathrm{M}$ amino acid mixture (the number of animals tested at each time point was $4,11,7,8$, and 9 , respectively), and (B) a $10 \mu \mathrm{M}$ amino acid mixture (the number of animals tested at each time point was $5,11,8,8$, and 7 , respectively). The magnitude of the EOG induced by the amino acid mixtures was significantly affected by the time to land adaptation (from the weighted linear model; $P<0.05$ ). The slope estimates from the weighted linear model (Table 1) indicate that the magnitude of the EOG to the $1 \mu \mathrm{M}$ and $10 \mu \mathrm{M}$ amino acid mixtures significantly increased with land adaptation time $(P<0.05)$. (C) The mean magnitude of the EOG to the volatile odorant mixture (the number of animals tested at each time point was $8,7,5,7$, and 11 , respectively). There was no significant relationship between the magnitude of the EOG response and land adaptation time (from the weighted linear model). The plot at each time point represents the mean \pm SE. 
the volatile odorant mixture. The magnitude of the EOG (mean \pm SE) at each time point was $570.24 \pm 89.44$, $778.70 \pm 154.40,695.84 \pm 90.18,480.29 \pm 217.57$, and $1014.98 \pm 236.31 \mu \mathrm{V}$, respectively. There was no significant relationship between the magnitude of the EOG response to the volatile odorants and land adaptation time (weighted linear model; $F_{1,36}=1.4726, P=0.2328$ ), in contrast to the results for the magnitude of the EOG response to the amino acid mixture.

No intercept estimates from the weighted linear model (Table 1) showed significant differences ( $t$-test). In other words, at $\mathrm{O} \mathrm{h}$ for newts kept in a water tank until just before dissection, there was no significant difference in the magnitudes of the EOG induced by the solutions we tested.

\section{DISCUSSION}

\section{Effects of land adaptation on EOG responses}

The statistical analysis indicated that the magnitude of the EOG induced by the amino acid mixture increased significantly with time to land adaptation (Fig. 2A, B). During the experiments, the olfactory epithelium was constantly perfused with test solutions, a condition similar to that experienced by animal olfaction in aquatic conditions, just after returning from terrestrial conditions. Amino acids are effective feeding stimuli. Several aquatic and semi-aquatic vertebrates, including newts, search for food in water by using their olfactory sense. Ferrer and Zimmer (2007) found in a behavioral study that the basic amino acid arginine was the most stimulatory compound for California newt (Taricha torosa) foraging activity, and estimated the response threshold for this activity to be $8.3 \times 10^{-1} \mu \mathrm{M}$. The magnitude of the EOG induced by the amino acid mixture increased in a dose-dependent manner after land adaptation (as determined by comparing the slope estimates at $1 \mu \mathrm{M}$ and $10 \mu \mathrm{M}$ with the $t$-test), while there was no significant difference in the magnitude of the EOG response to the $1 \mu \mathrm{M}$ and $10 \mu \mathrm{M}$ amino acid mixtures at $0 \mathrm{~h}$, as determined by comparing the intercept estimates ( $t$-test). The difference in the slopes estimated at $1 \mu \mathrm{M}$ and $10 \mu \mathrm{M}$ indicates that the effects of land adaptation increase in a dose-dependent manner. The reason is unknown; however, this may be due to change in the condition of the epithelial mucosa and/or fasting. In any case, land adaptation may have beneficial effects in allowing immediate foraging activity by newts returning to water.

Shibuya and Takagi (1963) reported that the newt olfactory epithelium does not respond at all to odorous vapors in water. In the present study, we used volatile odorants as odorous fluids, so that we could record EOG responses to volatile odorants under aquatic conditions (Figs. 1B, 2C; $0 \mathrm{~h}$ ). Recent electrophysiological studies on olfactory responses have used volatile odorants as test solutions (e.g., Kurahashi and Yau, 1993). Each molecule of a volatile or water-soluble odorant permeates the olfactory epithelial mucosa. Therefore, there is no difference in the EOG response between volatile vapors under terrestrial conditions and volatile fluids under laboratory conditions. Unlike the response to the mixture of amino acids, land adaptation had no significant effect on the magnitude of the EOG induced by volatile odorants (Fig. $2 \mathrm{C}$ ), indicating that the expected EOG response to odorous vapors is not influenced by land adaptation.

\section{Olfactory cilia and land adaptation}

It has been reported that aquatic and semi-aquatic vertebrates like fish (Muller and Marc, 1984) and Xenopus (Hansen et al., 1998) have ciliary and microvillar ORNs, but many species of amphibians, including newts, have only ciliary ORNs. Using whole-cell patch-clamping, Yamada and Nakatani (2001) showed that ciliary ORNs respond to both volatile odorants and amino acids in isolated ORNs of the newt.

Olfactory signal transduction takes place in the olfactory cilia (Kleene, 2008). ORs are localized on the plasma membrane of olfactory cilia (Buck and Axel, 1991), and olfactory transduction channels (CNG channels and $\mathrm{Ca}^{2+}$-activated $\mathrm{Cl}^{-}$channels) are present on the cilia (Takeuchi and Kurahashi, 2008). When newts are transferred from water to land, their olfactory cilia begin to grow. At $108 \mathrm{~h}$ after transfer, the length of the cilia reached $22 \mu \mathrm{m}$ (more than five times as long as those of newts in water), while the ciliary length of newts in water did not change (Shibuya and Takagi, 1963). The mechanism and function of the growth of olfactory cilia upon transfer from the aquatic to the terrestrial habitat is still unclear. Our present results suggest a correlation between the magnitude of EOG induced by amino acids and the growth of cilia caused by land adaptation. One possible explanation underlying the increase in sensitivity due to land adaptation, as measured by the EOG, is that the growth of cilia is accompanied by an increase in surface area, resulting in an increase in the number of ORs and consequently an increase in the magnitude of the EOG. However, Takeuchi and Kurahashi (2008) reported that the total ciliary currents do not differ much between cilia 15 and $30 \mu \mathrm{m}$ long. Moreover, our results indicate that volatile odorants induce no significant effects of land adaptation on the magnitude of EOG (Table 1). Therefore, the length of the cilia may not play an important role in the magnitude of the EOG response.

Our results suggest that the expression of amino acid receptors, but not of volatile odorant receptors, increases with land adaptation time. The reason for this selective expression of amino acid receptors during land adaptation is unknown. As we mentioned previously, amino acids are effective stimuli for feeding behavior. Terrestrial habitats may be unusual for adult newts, and the increase in olfactory sensitivity to amino acids may be important for the newts' survival on land.

\section{ACKNOWLEDGMENTS}

We thank Dr. Hiroyuki Mano for helpful advice on statistical analysis.

\section{REFERENCES}

Arzt AH, Silver WL, Mason JR, Clark L (1986) Olfactory responses of aquatic and terrestrial tiger salamanders to airborne and waterborne stimuli. J Comp Physiol A 158: 479-487

Buck L, Axel R (1991) A novel multigene family may encode odorant receptors: a molecular basis for odor recognition. Cell 65: 175187

Coman GJ, Sarac HZ, Fielder D, Thorne M (1996) Evaluation of crystalline amino acids, betaine and AMP as food attractants of the giant tiger prawn (Penaeus monodon). Comp Biochem Physiol A 113: 247-253

Copeland M (1913) The olfactory reactions of the spotted newt, 
Diemyctylus viridescens. J Anim Behav 3: 260-273

Ferrer RP, Zimmer RK (2007) Chemosensory reception, behavioral expression, and ecological interactions at multiple trophic levels. J Exp Biol 210: 1776-1785

Freitag J, Ludwig G, Andreini I, Rössler P, Breer H (1998) Olfactory receptors in aquatic and terrestrial vertebrates. J Comp Physiol A 183: $635-650$

Hansen A, Reiss JO, Gentry CL, Burd GD (1998) Ultrastructure of the olfactory organ in the clawed frog, Xenopus laevis, during larval development and metamorphosis. J Comp Neurol 398: 273-288

Hara TJ (1994) Olfaction and gustation in fish: an overview. Acta Physiol Scand 152: 207-217

Hara TJ (2006) Feeding behaviour in some teleosts is triggered by single amino acids primarily through olfaction. J Fish Biol 68: 810-825

Kleene SJ (2008) The electrochemical basis of odor transduction in vertebrate olfactory cilia. Chem Senses 33: 839-859

Kurahashi T (1990) The response induced by intracellular cyclic AMP in isolated olfactory receptor cells of the newt. J Physiol 430: 355-371

Kurahashi T, Yau KW (1993) Co-existence of cationic and chloride components in odrant-induced current of vertebrate olfactory receptor cells. Nature 363: 71-74

Mezler M, Fleischer J, Breer H (2001) Characteristic features and ligand specificity of the two olfactory receptor classes from Xenopus laevis. J Exp Biol 204: 2987-2997
Muller JF, Marc RE (1984) Three distinct morphological classes of receptors in fish olfactory organs. J Comp Neurol 222: 482-495

Nakamura T, Gold GH (1987) A cyclic nucleotide-gated conductance in olfactory receptor cilia. Nature 325: 442-444

Nakazawa H, Kaji S, Ishii S (2000) Oscillatory electric potential on the olfactory epithelium observed during the breeding migration period in the Japanese toad, Bufo japonicus. Zool Sci 17: 293300

Ottoson D (1956) Analysis of the electrical activity of the olfactory epithelium. Acta Physiol Scand 35 Suppl 122: 1-83

Quinn GP, Keough MJ (2002) Experimental design and data analysis for biologists. 1st ed, Cambridge University Press, Cambridge

Reese AM (1912) Food and chemical reactions of the spotted newt, Diemyctylus viridescens. J Anim Behav 2: 190-208

Shibuya T, Takagi SF (1963) Electrical response and growth of olfactory cilia of the olfactory epithelium of the newt in water and on land. J Gen Physiol 47: 71-82

Takeuchi H, Kurahashi T (2008) Distribution, amplification, and summation of cyclic nucleotide sensitivities within single olfactory sensory cilia. J Neurosci 28 : 766-775

Yamada H, Nakatani K (2001) Adenylate cyclase mediates olfactory transduction of amino acid responses in the newt. Zool Sci 18: 159-164

(Received August 10, 2009 / Accepted November 9, 2009) 